\section{HYDRATION IN MOLECULAR CRYSTALS}

A. L. Gillon $^{1}$ R. J. Davey ${ }^{1}$ N Feeder ${ }^{2}$ R Storey ${ }^{2}$

${ }^{1}$ UMIST Chemical Engineering P.O. Box 88 Sackville Street MANCHESTER M60 1QD UK ${ }^{2}$ Pharmaceutical R\&D, Pfizer Global R\&D, Sandwich, Kent, CT13 9NJ, UK

In the crystallization of molecular compounds from aqueous solutions the formation of hydrated crystals is common. Such materials can be problematic particularly in the pharmaceutical context since hydrated structures can possess adverse physical properties relating to solubility, stability, thermodynamic activity and bioavailability of the material. Thus there is a strong desire to understand more fully the combination of molecular and crystallographic factors which lead to the formation of crystalline hydrates.

One of the most commonly used classifications of crystalline hydrates has involved defining isolated site, channel or metal coordinated hydrates ${ }^{1}$ and acknowledges that the multidirectional hydrogen bonding capability of water is ideal for linking many small organic molecules into stable crystal structures. A previous study of organic hydrates in the Cambridge Structural Database $(\mathrm{CSD})^{2}$ analyzed the ratio of the number of hydrogen bond acceptor groups to donor groups. ${ }^{3}$ In this study the CSD has been used to investigate the detailed environment of the water molecules within organic hydrates. Six coordination states of water have been investigated, and the occurrences of certain donor and acceptor groups within these environments are reported. This analysis has been carried out on 3318 organic hydrates. The hydrogen bonding within specific systems, where both anhydrous and hydrate crystal structures are known, has been explored.

References

1. M. Falk and O. Knop, Water A Comprehensive Treatise, Vol. 2, Plenum Press, New York, 1973, 55.

2. F.H. Allen and O. Kennard, Chemical Design Automation News, 1993, 8(1), 31

3. G.R. Desiraju, J. Chem. Soc., Chem. Commun., 1991, 426

\section{Keywords: HYDRATES HYDROGEN BONDING CAMBRIDGE STRUCTURAL DATABASE}

\section{Acta Cryst. (2002). A58 (Supplement), C325 PATTERNS IN SATURATED HYDROGEN BONDING (SHB) J. H. Loehlin \\ Department of Chemistry Wellesley College WELLESLEY MA 02481 USA}

What do ice, ammonium chloride and piperazine have in common? In the past decade, Ermer \& Eling (J.Chem.Soc.Perkin Trans. 2, 1995, 925-944), we (Acta Cryst. B54, 1998, 695-704), and a few others have studied the complementary 1:1 alcohol/amine compounds in which the number of hydrogen bond donors exactly matches the number of acceptors.

Systematically extending this SHB concept to other complementary donor/acceptor arrangements has led to the investigation of several new structural patterns in our own research, as well as examining a number of structures from the literature. We classify participating non-hydrogen SHB atoms in terms of their number of hydrogens (d) and lone pairs (a) with a label da da. The alcohol amines are denoted 1221 and ice 2222 .

Our search for other examples led to the determination of piperazines crystal structure - an almost ideal 1111 material. Considering coordination numbers up to four, the number of simple binary SHB categories using this method is limited to eight, with known materials belonging to at least five of these. Are there stable materials in the remaining three categories (31 13, 3003 \& 20 02)? We have used Etters Graph Set (GS) approach to further describe the observed patterns in each category. This analysis illustrates the utility as well as some limitations of the GS model. Two or three-dimensional arrays involving identical hydrogen bonds, as in piperazine or ice, demonstrate problems somewhat analogous to those of describing a molecule of graphite or of diamond.

\section{Keywords: HYDROGEN BONDING PATTERNS GRAPH SETS}

Acta Cryst. (2002). A58 (Supplement), C325

STACKING ALTERNATIVES GENERATED BY THE EFFECT OF ANTIDROMIC RINGS IN CLOSE PACKING PATTERNS A. Kalman $^{1}$ Gy. Argay ${ }^{1}$ L. Fabian ${ }^{1}$ G. Bernath ${ }^{2}$ Zs. Gyarmati ${ }^{2}$

${ }^{1}$ Chemical Research Center Hungarian Academy of Sciences Institute of Chemistry Pob 17 BUDAPEST H-1525 HUNGARY ${ }^{2}$ Institute of Pharmaceutical Chemistry, University of Szeged, Hungary

Recently, our research - termed as combinatorial crystal chemistry - on the possible forms of supramolecular self-assembly of two vicinal functions $\mathrm{X}$ and $\mathrm{COX}\left(\mathrm{X}=\mathrm{OH}, \mathrm{NH}_{2}\right)$ linked with cis or trans orientation to alicyclic molecules of different size and substituents, lead to the following conclusion: The lateral and linear combinations of $\mathrm{H}$-bonded dimers (with different symmetries) assembled either antiparallel or parallel mode can be classified into 8 basic patterns of close packing. 3 of them include 18-membered antidromic (AD) rings, which (may) generate significant total dipole moment. Since dipoles must cancel out over the whole crystal, antiparallel stacking of the layers is compulsory. In cis-4-tert-butyl-2-hydroxy-1-cyclopentanecarboxylic acid the polar layers (space group (sg) $P n$ ) are rotated upon each other by screw axes. In this array the screw axes are perpendicular to, whereas in trans-2-hydroxy-1cycloheptanecarboxylic acid they are parallel with the best planes of the AD rings. In the former case $\mathrm{sg}$ is $P 2_{1} / n$, while in the latter case the antiparallel layer stacking corresponds to sg $P c a 2_{1}$. The molecules of their analogous carboxamide derivatives build up a pattern of parallel helices of the enantiomers. The helices are related by glide panes. In both structures the third hydrogen bond, formed by the carboxamide group, accounts for the AD rings. In trans-2-hydroxy-1-cyclopentanecarboxamide the effect of AD rings seems to be subdued by analogous homodromic rings ( $\mathrm{sg} P n a 2_{1}$ ), whereas in the cycloheptane homologue the overall dipole moment is cancelled out by antiparallel columns of the parallel helices, which results in sg Pbca.

\section{Keywords: SUPRAMOLECULAR CHEMISTRY HYDROGEN BONDS} LAYER STACKING

\section{Acta Cryst. (2002). A58 (Supplement), C325
MOLECULAR SELF-ASSEMBLING OF NITRO DERIVATIVES OF 2- OXO-1-NAPHTHALDIMINE VIA C-H'O INTERMOLECULAR HYDROGEN BONDS \\ G. Pavlovic $^{1}$ Z. Popovic ${ }^{2}$ V. Roje I. Leban $^{3}$}

${ }^{1}$ Faculty of Textile Technology, University of Zagreb Pierottijeva 6 ZAGREB 10000 CROATIA ${ }^{2}$ Laboratory of General and Inorganic Chemistry, Faculty of Science, University of Zagreb, Ul. Kralja Zvonimira 8, HR-10000 Zagreb,

Croatia ${ }^{3}$ Laboratory of Inorganic Chemistry, Faculty of Chemistry and Chemical Technology, University of Ljubljana, P.O. Box 537, 1001 Ljubljana, Slovenia

In the context of our investigation of Schiff bases metal complexes, Schiff bases of the type $\mathrm{Ar}-\mathrm{CH}=\mathrm{N}-\mathrm{Ar}$ derived from 2-hydroxy-1-naphthaldehyde and m-nitro (1) and p-nitro aniline (2) are prepared by template condensation. Despite their excellent Lewis basicity, Schiff bases derived from o-hydroxy aromatic aldehydes also perform interesting chromic behaviour, which is dependent on the tautomerization, molecular conformation, intra- and intermolecular hydrogen bonding. Compounds 1 and 2 exist in the solid state as the cis-quinoid (or ketoamino) tautomers characterized by strong, resonance assisted hydrogen bond of the $\mathrm{N}-\mathrm{H}{ }^{\circ} \mathrm{O}$ type $\left(\mathrm{N}^{\cdots} \mathrm{O}\right.$ distance is $2.554(2)$ and 2.555(5) $\AA$ in 1 and 2, respectively). The structure of 1 is a new polymorph since enolimino tautomer of 1 is already known (Yeap et al., Z. Kristallogr. NCS 213 (1998) 489-490). The molecule of 2 is almost planar, while the planarity is not so pronounced in the structure of 1 (dihedral angles between naphthaldiminato and nitro-phenyl rings are $4.6(2)$ and $6.6(1)^{\circ}$, respectively). The molecules are assembled using weak $\mathrm{C}-\mathrm{H} \cdots \mathrm{O}$ intermolecular hydrogen bonds between nitro oxygen atom and $\mathrm{C}$ - $\mathrm{H}$ phenyl group $(\mathrm{C} \cdots \mathrm{O}$ distances are $3.448(2)$ and $3.471(8) \AA$ in 1 and 2, respectively), but the self-assembly patterns are different, i.e. the meta position of $\mathrm{NO}_{2}$ group in 1 enables dimerization into 10 -membered discrete centrosymmetrical dimers, while in 2 the $\mathrm{p}-\mathrm{NO}_{2}$ group is too far away to enable the same mode of molecular assembling. Thus, the molecules of 2 are linked into infinite chains spreading in ac plane.

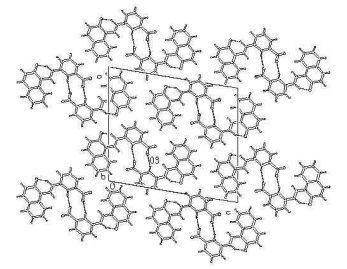

Keywords: KETOAMINO/ENOLIMINO- TAUTOMERS, C-H...O INTERMOLECULAR HYDROGEN BONDS, POLYMORPHISM 